\title{
Composição e variação sazonal do óleo essencial de Myrcia obtecta (O. Berg) Kiaersk. var. obtecta, Myrtaceae
}

\author{
Maria Élida Alves Stefanello, , ${ }^{, 1}$ Armando Carlos Cervi, ${ }^{2}$ Alberto Wisniewski Jr, ${ }^{3}$ \\ Edésio Luiz Simionatto ${ }^{3}$
}

\author{
${ }^{1}$ Departamento de Química, UFPR, Caixa Postal 19081, 81531-990 Curitiba-PR, Brasil \\ ${ }^{2}$ Departamento de Botânica, UFPR, Caixa Postal 19041, 81531-990 Curitiba- PR, Brasil \\ ${ }^{3}$ Instituto de Pesquisas Tecnológicas, Fundação Universidade de Blumenau, Rua Araçatuba 83, \\ 89030-080 Blumenau-SC, Brasil.
}

\begin{abstract}
RESUMO: Os óleos essenciais, isolados por hidrodestilação de folhas e flores de Myrcia obtecta (O. Berg) Kiaersk. var. obtecta, Myrtaceae, coletadas a cada dois meses em Curitiba, Paraná, durante o período de outubro/2005 a agosto/2006, foram analisados por CG e CG/EM. Os óleos das folhas foram caracterizados pelo elevado conteúdo de sesquiterpenos cíclicos (cerca de 70\%) e quantidades variáveis de monoterpenos (5,6-16,7\%), compostos alifáticos $(0,4-5,5 \%)$ e salicilato de metila (0,1-1,5\%). Foram identificados 55 componentes, sendo que trans-calameneno (17,0$29,3 \%$ ) foi o componente majoritário em todas as amostras. Não houve mudanças significativas na composição, excepto em outubro (floração) quando $\alpha$-terpineol e trans-calameneno atingiram os maiores níveis (11,2\% e 29,3\%, respectivamente). O óleo das flores contém salicilato de metila (89\%) como constituinte majoritário.
\end{abstract}

Unitermos: Myrcia obtecta, Myrtaceae, óleo essential, variação sazonal, trans-calameneno, salicilato de metila.

\begin{abstract}
Composition and seasonal variation of essential oils of Myrcia obtecta (O. Berg) Kiaersk. var. obtecta, Myrtaceae". The essential oils isolated by hydrodistillation from leaves and flowers of Myrcia obtecta (O. Berg) Kiaersk. var. obtecta, Myrtaceae, collected bimonthly in Curitiba, Paraná state, Brazil, from October/2005 to August/2006 were analyzed by capillary GC and GC/MS. Fifty-five components were identified in the leaf oils, which were characterized by predominance of cyclic sesquiterpenes (around 70\%) and variable amounts of monoterpenes (5.6$16.7 \%)$, aliphatic compounds (0.4-5.5\%) and methyl salicylate (0.1-1.5\%). The major component in all samples was trans-calamenene (17.0-29.3\%). The composition did not show significant seasonal variation, except in October (flowering) when $\alpha$-terpineol and trans-calamenene peaked $(11.2 \%$ and $29.3 \%$, respectively). The flower oil was characterized by high content of methyl salicylate ( $89 \%)$.
\end{abstract}

Keywords: Myrcia obtecta, Myrtaceae, essential oil, seazonal variation, trans-calamenene, methyl salicylate.

\section{INTRODUÇÃO}

A família Myrtaceae está representada no Brasil por 23 gêneros e cerca de mil espécies, sendo o gênero Myrcia um dos mais importantes, com aproximadamente quatrocentas espécies distribuídas sobre todo o território brasileiro (Landrum \& Kawasaki, 1997). Myrcia obtecta (O. Berg) Kiaersk., Myrtaceae, conhecida como guamirimbranco ou cambuí, é um arbusto ou árvore encontrada desde a Bahia até o Rio Grande do Sul. É particularmente abundante na região Sul do Brasil, ocorrendo na orla dos capões ou na submata dos pinhais. Legrand \& Klein (1969) reconhecem a existência de duas variedades: obtecta e alternifolia. A variedade principal (obtecta) apresenta folhas maiores e maior área de dispersão. Não foram encontrados registros de uso desta espécie na medicina popular.

Estudos prévios realizados com espécies de Myrcia mostraram a presença de monoterpenos, sesquiterpenos, compostos aromáticos e alifáticos nos óleos essenciais. Para a maioria das espécies estudadas a fração sesquiterpênica foi predominante (Cerqueira et al., 2007, 2009; Cole et al., 2008; Stefanello et al., 2007; Limberger et al., 2004; Zoghbi et al., 2003; Henriques et al., 1997). A composição do óleo essencial das folhas de M. obtecta (variedade não identificada) foi determinada anteriormente para um exemplar coletado no estado do Rio Grande do Sul (Limberger et al., 2004). Entretanto, a larga dispersão desta espécie e a existência de variedades botânicas podem resultar em considerável variação na 
composição do óleo. Além disso, o estudo foi limitado às folhas e a uma época de coleta. Por estes motivos, o presente trabalho teve como objetivos estudar a composição do óleo essencial de folhas e flores de Myrcia obtecta var. obtecta do Paraná e avaliar a ocorrência de variações sazonais no óleo das folhas.

\section{MATERIAL E MÉTODOS}

\section{Material vegetal}

Folhas e flores de Myrcia obtecta (O. Berg) Kiaersk. var. obtecta, Myrtaceae, foram coletadas de três exemplares, crescendo em um raio de $200 \mathrm{~m}$, em uma área particular em Curitiba-PR, Brasil. As coletas de folhas foram realizadas a cada dois meses, durante o período de outubro/2005 a agosto/2006, sempre no $15^{\circ}$ dia do mês pela manhã. As flores foram coletadas logo após a antese em outubro/2005. Uma exsicata foi depositada no herbário da Universidade Federal do Paraná (UPCB 60504).

\section{Obtenção dos óleos essenciais}

As folhas frescas dos três exemplares foram misturadas de modo a se obter um perfil químico do grupo. Em seguida, foram separadas duas porções de $100 \mathrm{~g}$, que foram submetidas, separadamente, a hidrodestilação, em um aparelho tipo Clevenger durante duas horas. $\mathrm{O}$ hidrolato foi extraído com éter dietílico, a fase orgânica foi separada e secada com sulfato de sódio. O solvente foi removido por aplicação de vácuo e o óleo conservado em congelador até a análise. Deste modo foram obtidos dois óleos para cada coleta. As flores foram tratadas do mesmo modo. O rendimento dos óleos foi calculado relacionandose a massa de óleo obtida e a massa de material vegetal utilizado na extração.

\section{Análise Química}

A análise quantitativa foi realizada em um cromatógrafo gasoso Shimadzu GC-17A, equipado com uma coluna capilar Durabond-DB5 (30 m x 0,25 $\mathrm{mm} \times 0,25 \mu \mathrm{m})$ e um detector de ionização de chama. A temperatura inicial foi mantida a $60{ }^{\circ} \mathrm{C}$ por $3 \mathrm{~min}$., em seguida programada até $250{ }^{\circ} \mathrm{C}$ a $5{ }^{\circ} \mathrm{C} / \mathrm{min}$ e finalmente mantida a $250{ }^{\circ} \mathrm{C}$ por $15 \mathrm{~min}$. $\mathrm{O}$ gás de arraste foi hélio, usado a uma pressão constante de $80 \mathrm{Kpa}$ e vazão constante de $1 \mathrm{~mL} / \mathrm{min}$. A quantificação dos componentes foi obtida por integração eletrônica, sem correção da resposta do detector.

A análise qualitativa foi realizada em um cromatógrafo gasoso Varian CP-800, acoplado a um espectrômetro de massas Saturn 2000, equipado com uma coluna capilar CP-Sil $8 \mathrm{CB}$ (30 m x 0,25 mm x 0,25 $\mu \mathrm{m})$. As condições de operação foram as mesmas descritas acima. A identificação dos componentes do óleo foi baseada no índice de retenção linear calculado em relação aos tempos de retenção de uma série homóloga de $n$-alcanos (IR) e no padrão de fragmentação observado nos espectros de massas, por comparação destes com dados da literatura (Adams, 2007) e da espectroteca Nist 98. Adicionalmente, limoneno e eugenol foram confirmados por comparação com padrões (Sigma-Aldrich).

Espectros de $\mathrm{RMN}{ }^{1} \mathrm{H}$ e ${ }^{13} \mathrm{C}$ foram registrados para alguns óleos, em um espectrômetro Brucker AC200, operando a $200 \mathrm{MHz}$ para o hidrogênio e $50 \mathrm{MHz}$ para o carbono, utilizando-se clorofórmio deuterado como solvente e TMS como referência interna.

\section{RESULTADOS E DISCUSSÃO}

As folhas forneceram óleos incolores com rendimento médio de $0,01 \%$. Não houve diferença significativa no rendimento entre as diversas coletas. Foram identificados no total 55 componentes (cerca de $80 \%$ do total do óleo). Os sesquiterpenos foram a classe predominante, representando aproximadamente $70 \%$ dos compostos identificados em todas as amostras. Também foram encontrados monoterpenos, compostos aromáticos e alifáticos (Tabela 1).

O constituinte principal em todas as amostras de folhas foi o sesquiterpeno trans-calameneno. Este composto representou quase $30 \%$ da amostra do mês de outubro e manteve-se em torno de $20 \%$ nos demais meses. A identidade desta substância foi confirmada através de análise do espectro de $\mathrm{RMN}{ }^{1} \mathrm{H}$ do óleo (amostra de outubro) e comparação com dados da literatura (Nakashima et al., 2002). Outro componente que se destacou foi o monoterpeno $\alpha$-terpineol, que mostrou um teor significativo $(11,2 \%)$ apenas na amostra de outubro. Todos os demais constituintes foram minoritários (abaixo de 8\%). Essa composição difere daquela encontrada em estudo anterior (Limberger et al., 2004) que continha Ar-curcumeno como constituinte majoritário (19\%) e quantidades significativas de $\beta$-bisaboleno (8,5\%), $\alpha$-copaeno $(8,0 \%)$ e $\alpha$-pineno $(7,2 \%)$. Dentre estes compostos apenas $\alpha$-copaeno $(1,0-$ $1,5 \%$ ) foi encontrado no presente estudo.

Excluindo-se a amostra de outubro (quando a árvore estava florindo), as demais não mostraram variações significativas na composição do óleo das folhas. Portanto, para o grupo estudado o período de floração exerce influência sobre os constituintes do óleo das folhas. Essa situação é bastante comum, tendo sido observada anteriormente em várias espécies (Figueiredo et al., 2008; Mirjalili et al., 2006). Dentre as espécies de Myrcia já estudadas, Myrcia obtecta (O. Berg) Kiaersk.var. obtecta, Myrtaceae, e M. salzmannii tiveram análises de variação sazonal (Cerqueira et al., 2007, 2009), mas nestes casos as alterações observadas não puderam ser associadas com o período de floração.

As flores forneceram um óleo incolor constituído quase exclusivamente de salicilato de metila $(88,2 \%)$. 
Outros compostos aromáticos (álcool benzílico, feniletanol e eugenol) que não haviam sido detectados nas folhas também foram observados juntamente com alguns terpenoides. A presença de salicilato de metila foi confirmada através da análise de espectros de RMN ${ }^{1} \mathrm{H}$ e ${ }^{13} \mathrm{C}$ e comparação com dados da literatura (Nébié et al., 2004). Diferenças entre a composição do óleo essencial de folhas e flores são esperadas, uma vez que o aroma emitido pelas flores está associado à atração de polinizadores específicos (Cseke et al., 2007).

Tabela 1. Composição química percentual (\%)a dos óleos essenciais de folhas e flores de M. obtecta

\begin{tabular}{|c|c|c|c|c|c|c|c|c|}
\hline \multirow[t]{2}{*}{ Composto } & \multirow[t]{2}{*}{ IR } & \multicolumn{6}{|c|}{ Folhas } & \multirow{2}{*}{$\begin{array}{c}\text { Flores } \\
\text { Out }\end{array}$} \\
\hline & & Out & Dez & Fev & Abr & Jun & Ago & \\
\hline 2-E-hexanal & 860 & 0,1 & 2,2 & 0,9 & 1,1 & 1,7 & 3,1 & 0,1 \\
\hline mirceno & 991 & 0,3 & 0,7 & 0,2 & 0,3 & 0,4 & 0,8 & - \\
\hline$p$-cimeno & 1025 & 0,2 & - & - & 0,2 & 0,2 & 0,2 & - \\
\hline limoneno & 1027 & 0,5 & 1,5 & 1,4 & 1,0 & 1,7 & 2,1 & - \\
\hline álcool benzílico & 1037 & - & - & - & - & - & - & 0,9 \\
\hline$\gamma$-terpineno & 1058 & 0,1 & 0,3 & - & - & - & - & - \\
\hline isoterpinoleno & 1085 & 0,1 & - & - & 0,2 & 0,4 & - & 0,2 \\
\hline linalol & 1102 & - & - & - & 0,5 & 0,5 & 0,6 & \\
\hline feniletanol & 1116 & - & - & - & - & - & - & 1,5 \\
\hline exo-fenchol & 1120 & 0,1 & - & 0,2 & 0,2 & 0,1 & - & - \\
\hline borneol & 1173 & 0,1 & 0,1 & 0,1 & 0,2 & - & 0,1 & - \\
\hline terpinen-4-ol & 1182 & 4,2 & 0,9 & 2,5 & 2,2 & 4,0 & 3,7 & - \\
\hline salicilato de metila & 1194 & 1,5 & 0,1 & 0,1 & 0,1 & 0,1 & 0,2 & 88,2 \\
\hline$\alpha$-terpineol & 1197 & 11,2 & 2,2 & 4,6 & 4,3 & 5,2 & 3,9 & - \\
\hline eugenol & 1357 & - & - & - & - & - & - & 1,9 \\
\hline ciclosativeno & 1368 & - & - & - & 0,2 & - & - & - \\
\hline$\alpha$-copaeno & 1375 & 1,5 & 1,1 & 1,4 & 1,5 & 1,5 & 1,0 & - \\
\hline$\beta$-bourboneno & 1383 & - & - & - & 0,1 & - & - & - \\
\hline$\beta$-cubebeno & 1388 & - & 0,3 & - & 0,3 & - & - & - \\
\hline$\alpha$-gurjuneno & 1406 & - & 0,4 & - & 0,5 & 0,2 & 0,2 & - \\
\hline$\beta$-cariofileno & 1418 & 2,0 & 2,5 & 1,5 & 3,3 & 3,4 & 2,4 & - \\
\hline$\alpha$-guaieno & 1434 & 5,8 & 5,3 & 7,8 & 7,3 & 5,3 & 4,2 & 0,8 \\
\hline aromadendreno & 1438 & 0,1 & 0,6 & 0,4 & 0,5 & 0,5 & 0,5 & - \\
\hline 6,9-guaiadieno & 1440 & 0,1 & 0,3 & 0,2 & 0,3 & 0,2 & 0,2 & - \\
\hline cis-muurola-3,5-dieno & 1448 & - & 1,2 & 2,1 & 1,0 & 0,8 & 1,0 & - \\
\hline$\alpha$-humuleno & 1454 & 4,6 & 3,9 & 2,6 & 4,7 & 4,8 & 3,4 & - \\
\hline allo-aromadendreno & 1459 & - & 0,8 & 0,8 & 0,8 & 1,0 & 0,7 & - \\
\hline cadina-1(6),4-dieno & 1471 & 2,4 & 4,1 & 4,6 & 5,4 & 3,2 & 1,9 & - \\
\hline$\gamma$-muuroleno & 1478 & 1,4 & 1,1 & 1,0 & 0,9 & 0,9 & 0,6 & - \\
\hline$\gamma$-himachaleno & 1482 & 2,4 & 2,0 & 1,6 & 1,8 & 1,8 & 1,3 & - \\
\hline$\beta$-selineno & 1488 & 0,1 & 1,8 & 2,1 & 2,1 & 2,1 & 1,8 & - \\
\hline$\delta$-selineno & 1490 & 0,1 & 2,1 & 1,0 & 1,9 & 1,6 & 1,0 & - \\
\hline$\alpha$-selineno & 1495 & 0,1 & 1,3 & 1,7 & 1,5 & 1,4 & 1,1 & - \\
\hline$\beta$-himachaleno & 1499 & 0,5 & 3,2 & 1,8 & 0,1 & 0,2 & 0,1 & - \\
\hline$\gamma$-cadineno & 1513 & - & 0,6 & - & 0,5 & 0,5 & 0,5 & - \\
\hline
\end{tabular}

flores de M. obtecta var. obtecta diferem consideravelmente entre si, sendo o primeiro constituído principalmente por sesquiterpenos e o segundo por salicilato de metila. As flores podem ser consideradas uma nova fonte natural de salicilato de metila, pois o seu percentual é de quase $90 \%$. A composição do óleo das folhas sofre influência da floração e difere daquele encontrado em exemplares crescendo no Rio Grande do Sul. 


\begin{tabular}{|c|c|c|c|c|c|c|c|c|}
\hline$\delta$-cadineno & 1524 & 1,2 & 3,1 & 3,1 & 2,8 & 2,3 & 1,9 & - \\
\hline trans-calameneno & 1522 & 29,3 & 17,3 & 18,0 & 17,0 & 22,0 & 20,0 & 2,4 \\
\hline trans-cadina-1,4-dieno & 1532 & 4,8 & 3,2 & 3,1 & 2,8 & 2,8 & 2,1 & - \\
\hline$\alpha$-calacoreno & 1535 & - & 0,4 & 0,7 & 0,6 & 1,0 & 0,9 & - \\
\hline$\beta$-calacoreno & 1541 & - & 0,4 & 0,6 & 0,7 & 0,8 & 1,0 & - \\
\hline espatulenol & 1577 & - & 0,5 & - & 0,7 & 0,3 & 0,4 & - \\
\hline óxido de cariofileno & 1581 & 3,4 & 1,4 & 3,0 & 2,4 & 1,7 & 2,0 & - \\
\hline globulol & 1586 & 0,3 & 1,2 & 1,2 & 1,0 & 0,6 & 0,8 & - \\
\hline viridiflorol & 1594 & - & 0,6 & 1,0 & 0,8 & 0,7 & 1,2 & 0,8 \\
\hline cubeban-11-ol & 1597 & - & 1,2 & 1,2 & 0,9 & 0,6 & 0,6 & - \\
\hline guaiol & 1604 & - & 0,4 & 0,6 & 0,6 & 0,1 & 0,4 & 1,1 \\
\hline 5-epi-7-epi- $\alpha$-eudesmol & 1607 & - & 0,5 & - & 0,3 & - & 0,3 & - \\
\hline epóxido de humuleno II & 1610 & 1,9 & 1,5 & 3,2 & 1,9 & 1,3 & 1,9 & - \\
\hline óxido de $\beta$-himachaleno & 1613 & - & 0,6 & - & 0,5 & 0,3 & 0,6 & - \\
\hline junenol & 1622 & - & 0,5 & - & 0,8 & - & 0,6 & - \\
\hline 1-epi-cubenol & 1628 & 5,6 & 1,6 & 2,0 & 1,3 & 1,8 & 2,7 & - \\
\hline$\gamma$-eudesmol & 1633 & - & 0,7 & 1,1 & 0,9 & 0,3 & 0,6 & - \\
\hline$\alpha$-muurolol & 1643 & 3,7 & 2,0 & 2,1 & 1,6 & 1,6 & 1,7 & - \\
\hline cubenol & 1645 & 4,6 & 1,6 & 1,9 & 1,5 & 1,3 & 1,5 & - \\
\hline$\alpha$-cadinol & 1656 & 1,4 & 2,0 & 2,9 & 2,4 & 1,7 & 2,4 & - \\
\hline cadaleno & 1674 & - & 0,2 & - & 0,2 & 1,0 & 1,1 & - \\
\hline khusinol & 1679 & - & 0,4 & - & 0,4 & 0,2 & 0,2 & - \\
\hline amorfa-4:9-dien-14-al & 1703 & - & 0,6 & - & 0,2 & 0,2 & 0,9 & - \\
\hline Total identificado & & 95,6 & 82,5 & 86,3 & 87,3 & 86,3 & 82,4 & 97,9 \\
\hline Monoterpenos & & 16,7 & 5,7 & 9,0 & 9,1 & 12,5 & 11,4 & 0,2 \\
\hline Sesquiterpenos não oxigenados & & 56,4 & 57,0 & 56,1 & 58,6 & 58,3 & 47,8 & 3,2 \\
\hline Sesquiterpenos oxigenados & & 20,9 & 17,5 & 20,2 & 18,4 & 13,7 & 19,9 & 1,9 \\
\hline Compostos aromáticos & & 1,5 & 0,1 & 0,1 & 0,1 & 0,1 & 0,2 & 92,5 \\
\hline Compostos alifáticos & & 0,1 & 2,2 & 0,9 & 1,1 & 1,7 & 3,1 & 0,1 \\
\hline
\end{tabular}

a Os valores representam a média de duas análises. Compostos listados em ordem de eluição de uma coluna DB-5; amostras coletadas entre outubro/2005 a agosto/2006; IR: índice de retenção experimental médio; - composto não detectado.

\section{REFERÊNCIAS}

Adams RP 2007. Identification of Essential Oils Components by Gas chromatography/Mass Spectrometry, Allured Publ. Corp.,Carol Stream, IL.

Cerqueira MD, Souza-Neta L, Passos MGVM, Lima EO, Roque NF, Martins D, Guedes MLS, Cruz FG 2007. Seasonal variation and antimicrobial activity of Myrcia myrtifolia essential oils. J Braz Chem Soc 18: 998-1003.

Cerqueira MD, Marques EJ, Martins D, Roque NF, Cruz FG, Guedes MLS 2009. Variação sazonal da composição do óleo essencial de Myrcia salzmannii Berg. (Myrtaceae). Quim Nova 32: 1544-1548.

Cole RA, Haber WA, Setzer WN 2008. The leaf oil composition of Myrcia splendens from Monteverde, Costa Rica. J Essent Oil-Bear Plants 11: 41-44.

Cseke LJ, Kaufman PB, Kirakosyan A 2007. The biology of essential oils in the pollination of flowers. Nat Prod Commun 2: 1317-1336.
Figueiredo AC, Barroso JG, Pedro LG, Scheffer JJC 2008. Factors affecting secondary metabolite production in plants: volatile components and essential oils. Flavour Frag J 23: 213-226.

Henriques AT, Sobral M, Bridi R, Vérin P, Menut C, Lamaty G, Bessière JM 1997. Essential oils from five southern brazilian species of Myrcia (Myrtaceae). J Essent Oil Res 9: 13-18.

Landrum LR, Kawasaki ML 1997. The genera of Myrtaceae in Brazil: an illustrated synoptic treatment and identification keys. Brittonia 49: 508-536.

Legrand CD, Klein RM 1969. Mirtáceas. In: Reitz R (org.). Flora Ilustrada Catarinense. Itajaí: Herbário Barbosa Rodrigues, p. 278-284.

Limberger RP, Sobral M, Henriques AT, Menut C, Bessière J 2004. Óleos voláteis de espécies de Myrcia nativas do Rio Grande do Sul. Quim Nova 27: 916-919.

Mirjalili MH, Salchi P, Sonboli A, Vata MM 2006. Essential oil variation of Salvia officinalis aerial parts during its 
phenological cycle. Chem Nat Compd 42: 19-23.

Nakashima K, Imoto M, Sono M, Tori M, Nagashima F, Asakawa Y 2002. Total synthesis of (-)-(7S,10R)-Calmenene and (-)-(7S,10R)-2-Hydroxycalamenene by use of a ringclosing metathesis reaction. A comparison of the cis-and trans-Isomers. Molecules 7: 517-527.

Nébié RHC, Yaméogo RT, Bélanger A, Sib FS 2004. Salicylate de méthyle, constituant unique de l'huile essentielle de l'écorce des racines de Securidaca longepedunculata du Burkina Faso. C R Chim 7: 1003-1006.

Stefanello MEA, Cervi AC, Wisniewski Jr A, Simionatto EL 2007. Essential oil composition of Myrcia laruotteana Camb. J Essent Oil Res 19: 466-467.

Zoghbi MGB, Andrade EHA, Silva MHL, Carreira LMM, Maia JGS 2003. Essential oils from three Myrcia species. Flavour Frag J 18: 421-424. 